\title{
Microfluidic Platform for the Isolation of Cancer- Cell Subpopulations based on Single-Cell Glycolysis
}

Claudia Zielke, ${ }^{1}$ Ching W. Pan, ${ }^{1}$ Adriana J. Gutierrez Ramirez, ${ }^{1}$ Cameron Feit, ${ }^{1}$ Chandler Dobson, ${ }^{1}$ Catherine Davidson, ${ }^{1}$ Brody Sandel, ${ }^{2}$ Paul Abbyad ${ }^{1 *}$

1 Department of Chemistry and Biochemistry, Santa Clara University, Santa Clara, CA, 95053, USA

2 Department of Biology, Santa Clara University, Santa Clara, CA, 95053, USA

* corresponding author: pabbyad@scu.edu

\section{Supplemental Information}

Table of Contents:

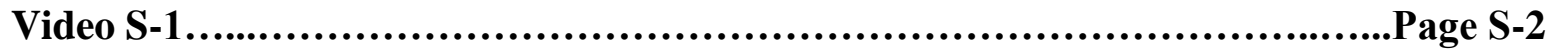

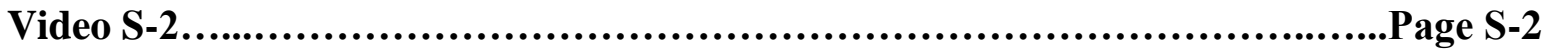

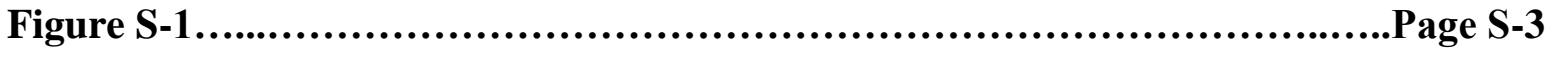

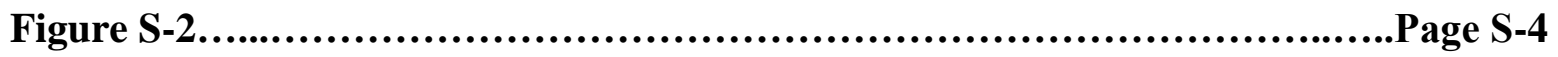

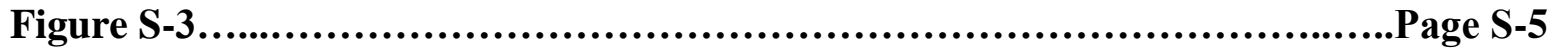

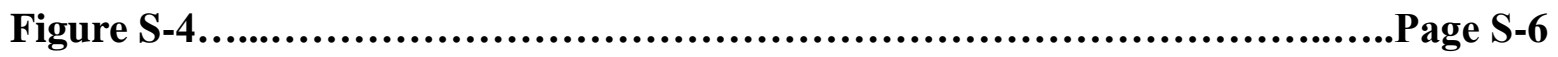

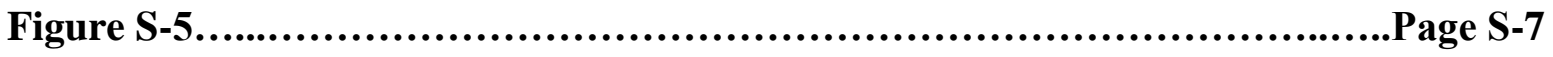

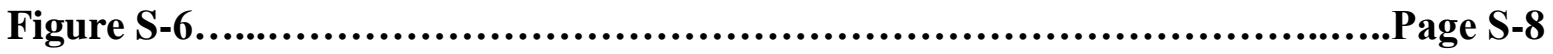

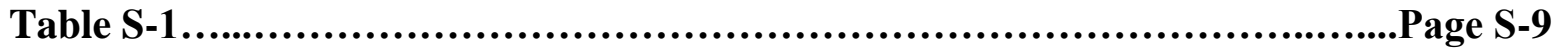




\section{Video Caption:}

Video S1. Selection of droplets of different pH containing K562 cells using SIFT. Video shows the end of incubator and sorting region.

Video S2. SIFT device sorting of hypoxia treated and control (normoxia) MDA-MB 231 cells. Video shows the end of incubator and sorting region. 


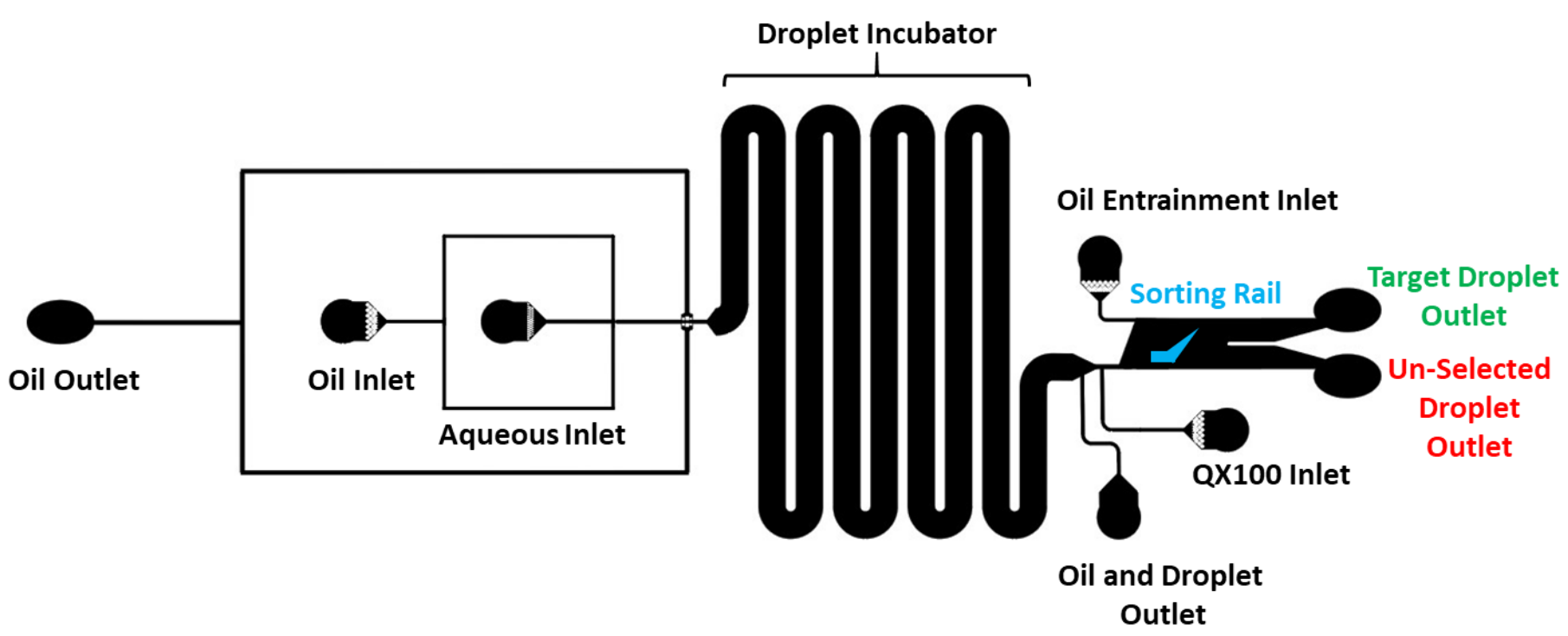

Supplemental Figure S1. SIFT device channel geometry. 


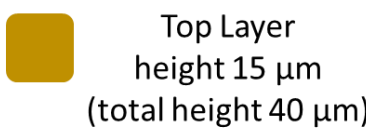

Bottom Layer

height $25 \mu \mathrm{m}$
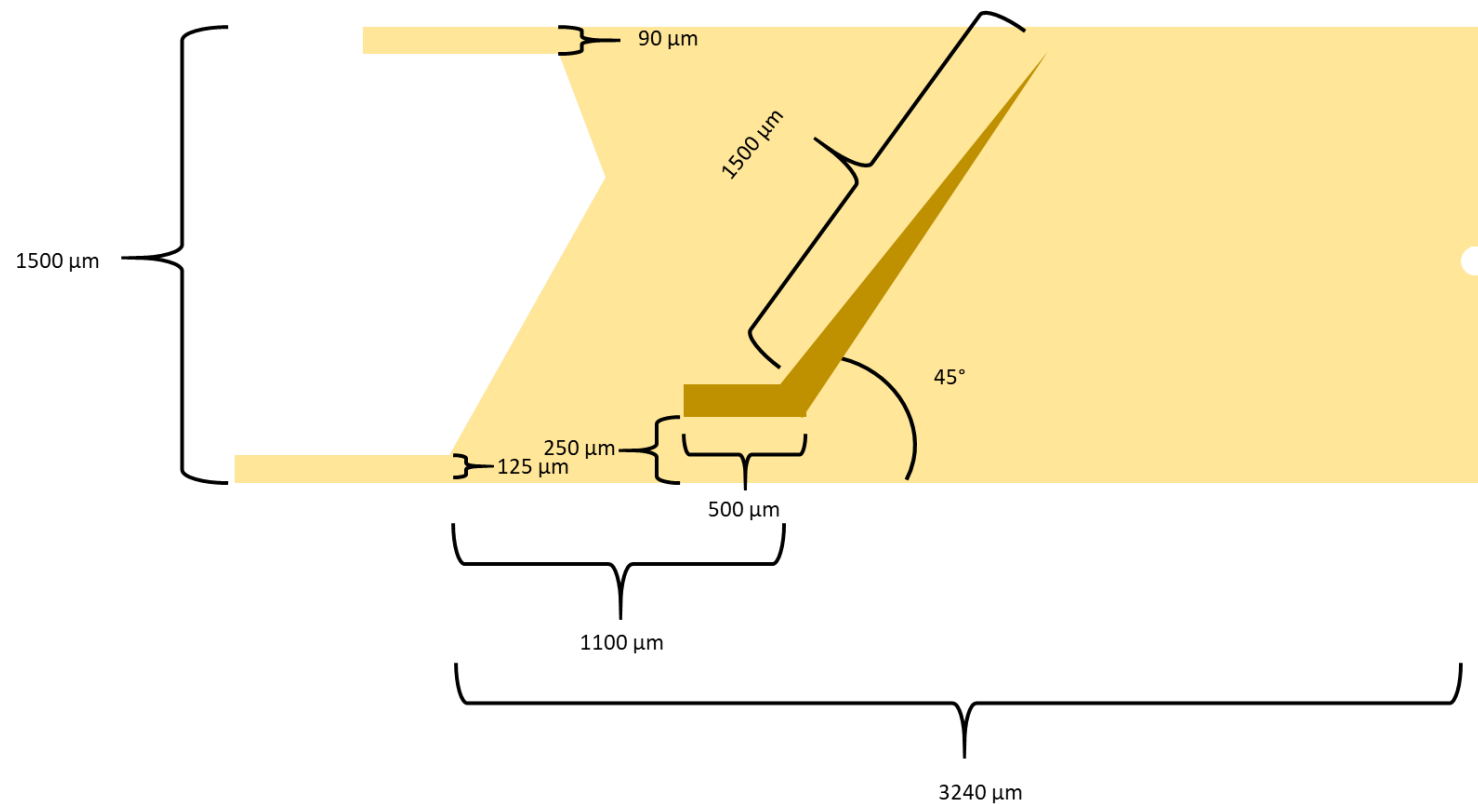

Supplemental Figure S2. Sorting rail dimensions and position. Exact position of rail is approximate as layers are positioned by eye. 


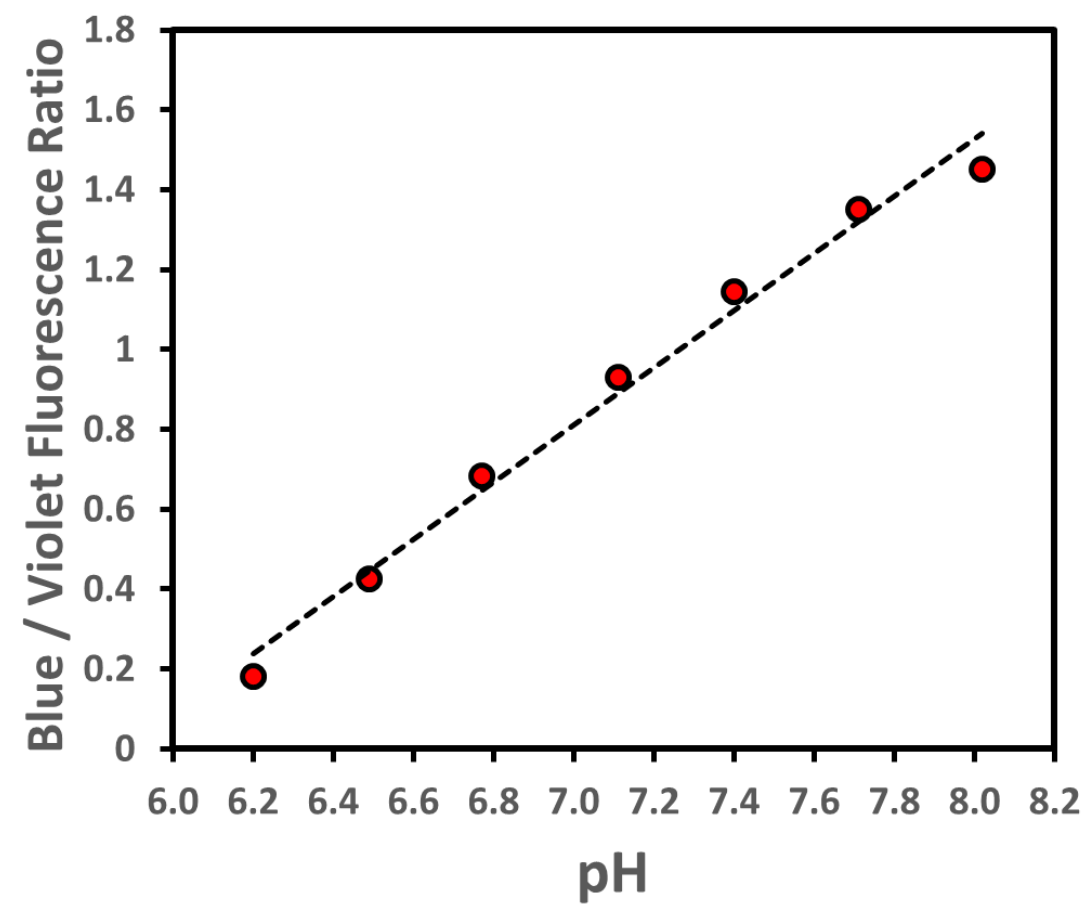

Supplemental Figure S3. Blue to violet fluorescence intensity ratio vs $\mathrm{pH}$ of droplets containing pyranine, a $\mathrm{pH}$ sensitive ratiometric fluorescent probe. Solutions of known $\mathrm{pH}$ were injected into the microfluidic device and droplets were analyzed for their normalized fluorescence intensity through excitation with blue $(440 \mathrm{~nm})$ and violet $(395 \mathrm{~nm})$ light. The calibration curve was obtained under the same conditions as cellular experiments. The slope of the calibration curve was found to be consistent from day to day, whereas the $y$-intersection was adjusted for each experiment using the known $\mathrm{pH}$ of empty droplets. 

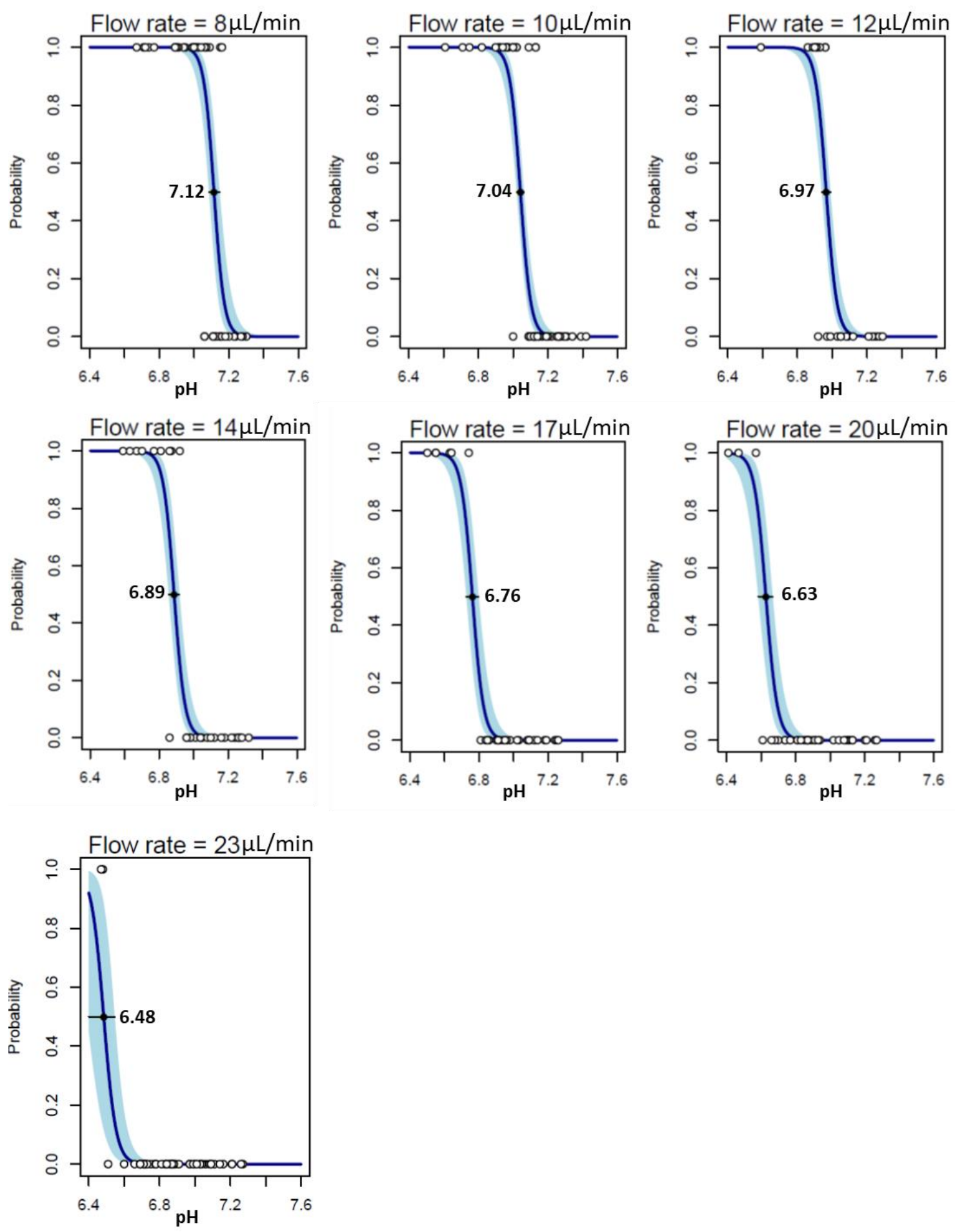

Supplemental Figure S4. A multiple logistic regression fit of binary selected/unselected data with $\mathrm{pH}$ at multiple flow rates. $\mathrm{pH}$ thresholds are indicated on graph and represent where there is equal probability that droplets are selected or unselected. The $95 \%$ confidence limit is indicated in light blue. 

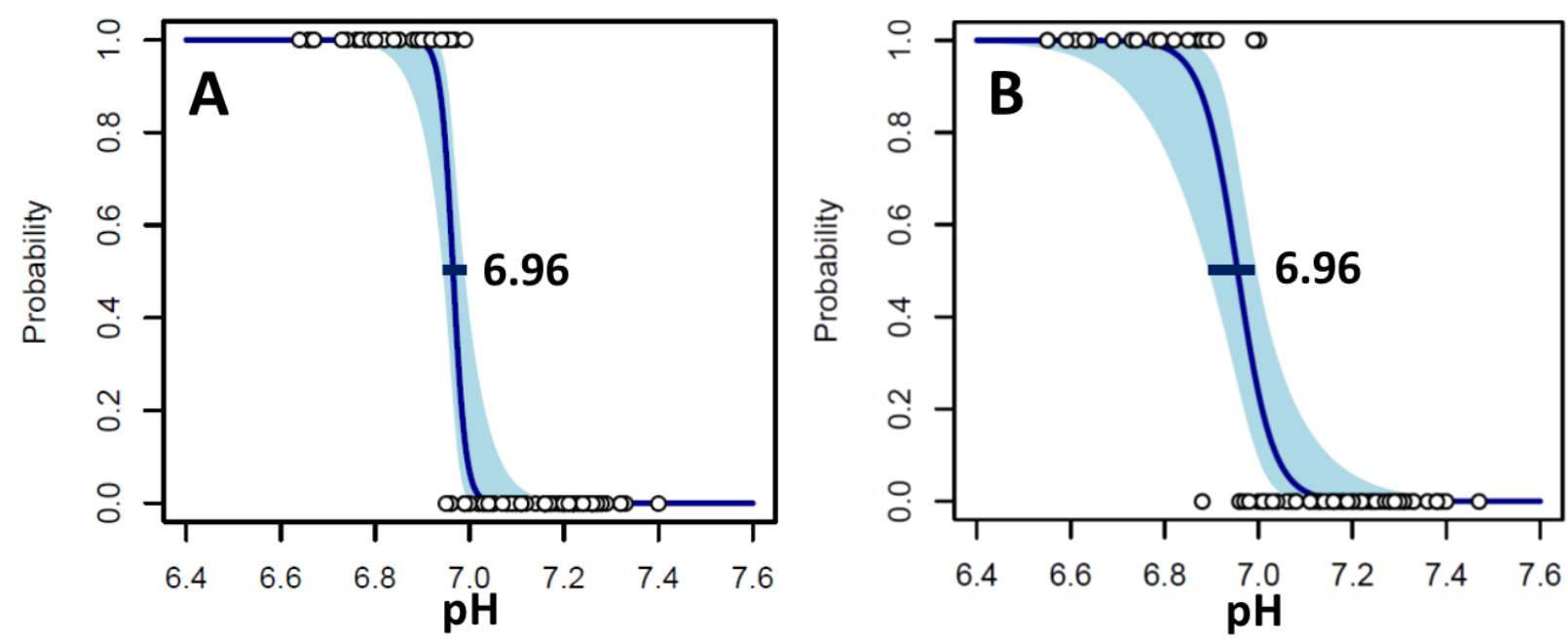

Supplemental Figure S5. A logistic regression fit of binary selected/unselected data with $\mathrm{pH}$ for A) hypoxia treated and control MDA-MB 231 cells and B) 2DG treated and control K562 cells. $\mathrm{pH}$ thresholds are indicated on graph and represent where there is equal probability that droplets are selected or unselected. The $95 \%$ confidence limit is indicated in light blue. 

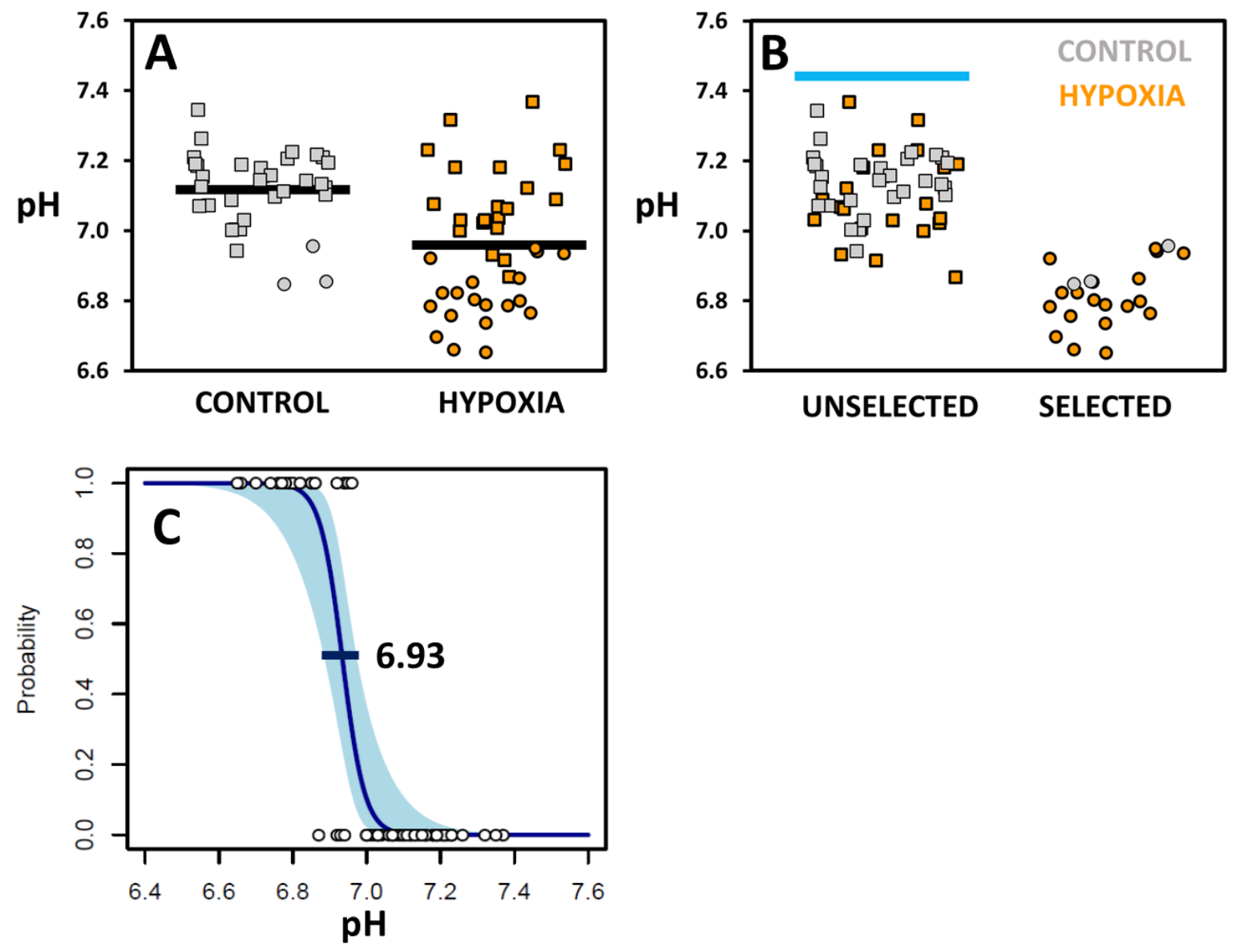

Supplemental Figure S6: pH of droplets containing either control (grey) or hypoxia treated (orange) K562 cells. Square markers annotate unselected, circular markers selected droplets. pH values were determined via a fluorescence intensity ratio. A) Droplets presented as control and hypoxia treated cell populations. Average $\mathrm{pH}$ values, indicated by a black line, are $7.12 \pm 0.02(\mathrm{~N}$ $=34)$ for control and $6.96 \pm 0.03(\mathrm{~N}=39)$ for hypoxia cells. B) Droplets presented as unselected and selected populations. Blue line indicates the $\mathrm{pH}$ of empty droplets $(\mathrm{pH} 7.40 \pm 0.04)$. The sorting of droplets leads to an enrichment of hypoxia treated cells from $54 \%(\mathrm{~N}=39)$ before sorting to $86 \%(\mathrm{~N}=19)$ of selected cells. C) The $\mathrm{pH}$ threshold of selection was determined by a logistic regression fit and was found to be $6.93 \pm 0.05$ (threshold $\pm 95 \%$ confidence interval). 
Supplemental Table S1. Typical flow parameters used in experiments. Channel geometry is provided below for reference. Negative flows below are opposite in direction to the main flow in the channel.

\begin{tabular}{|l|l|}
\hline Inlets and Outlets & Flow Rates $(\mu \mathrm{L} / \mathrm{min})$ \\
\hline Aqueous Inlet & $0.1-0.5$, most commonly 0.3 \\
\hline Oil Inlet & $3-5$, most commonly 3 \\
\hline QX100 Inlet & $2-5$ \\
\hline Oil Entrainment Inlet & $8-20$ \\
\hline Oil Outlet & -2.7 to -4.7 \\
\hline Oil and Droplet Outlet & -0.25 to -1 \\
\hline
\end{tabular}

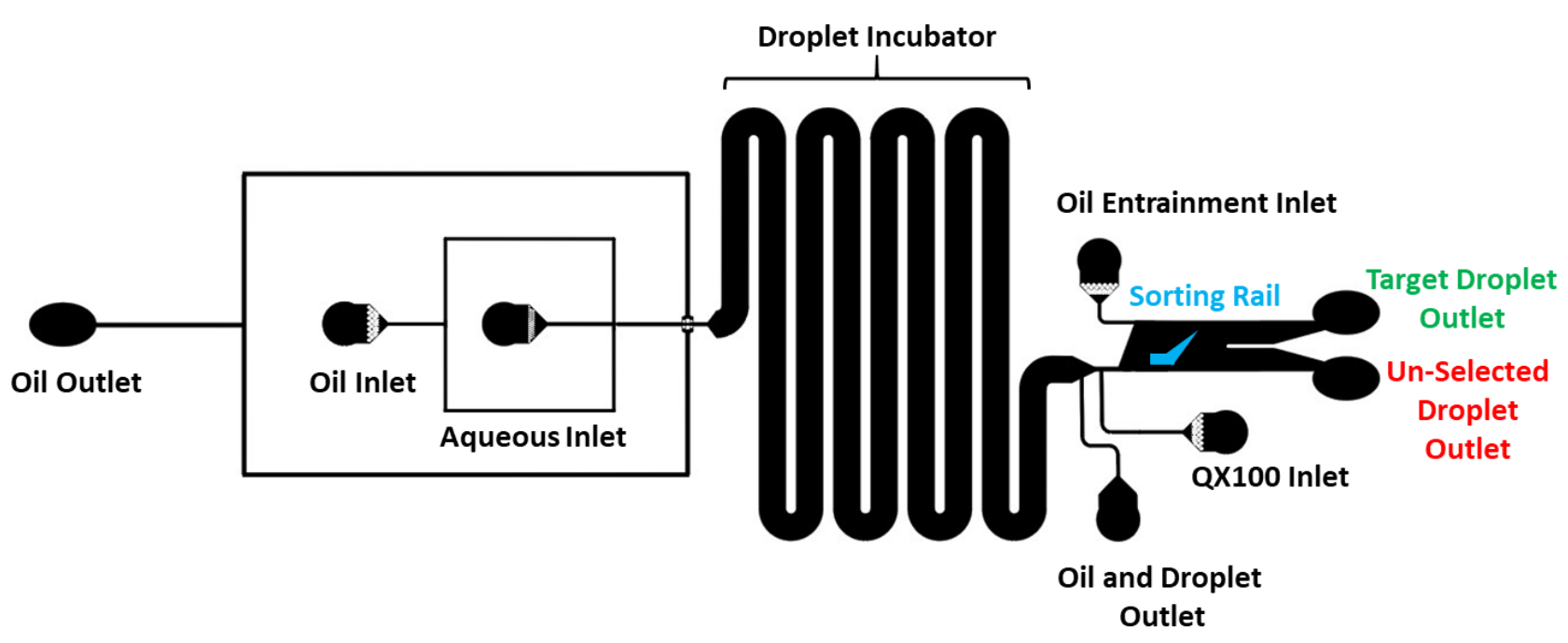

